\title{
RPE Cells Engulf Microvesicles Secreted by Degenerating Rod Photoreceptors
}

\author{
Philip Ropelewski and ํㅜoshikazu Imanishi
}

https://doi.org/10.1523/ENEURO.0507-19.2020

Department of Pharmacology, Case Western Reserve University School of Medicine, Cleveland, Ohio 44106

\section{Visual Abstract}

\section{Destiny of Mislocalized Rhodopsin}

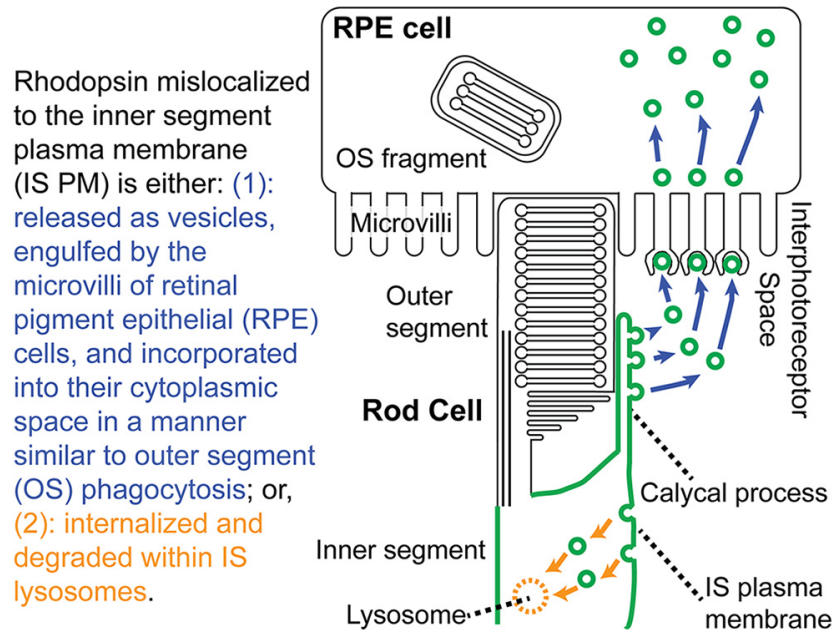

Rhodopsin is mislocalized to the inner segment plasma membrane (IS PM) in various blinding disorders including autosomal-dominant retinitis pigmentosa caused by class I rhodopsin mutations. In these disorders, rhodopsin-laden microvesicles are secreted into the extracellular milieu by afflicted photoreceptor cells. Using a Xenopus laevis model expressing class I mutant rhodopsin or $\mathrm{Na}^{+} / \mathrm{K}^{+}$-ATPase (NKA) fused to Dendra2, we fluorescently labeled the microvesicles and found retinal pigment epithelial (RPE) cells are capable of engulfing microvesicles containing rhodopsin. A unique sorting mechanism allows class I mutant rhodopsin, but not NKA, to be packaged into the microvesicles. Under normal physiological conditions, NKA is not shed as

\section{Significance Statement}

Rhodopsin mislocalization is a common cause of photoreceptor degeneration in inherited blinding disorders. In this study, we describe a novel mechanism of removing rhodopsin molecules from inner segment plasma membrane (IS PM). Mislocalized rhodopsin is packaged into microvesicles, which are secreted into the interphotoreceptor space and cleared via engulfment by retinal pigment epithelial (RPE) cells. While IS $\mathrm{PM}$-mislocalized rhodopsin is specifically packaged into microvesicles, $\mathrm{Na}^{+} / \mathrm{K}^{+}$-ATPase $\alpha$-subunit, an IS PM resident protein, was not sorted into vesicles under either pathologic or normal physiological conditions. Interaction between photoreceptor and RPE cells is critical for maintaining visual function, and its alteration can lead to compromised vision. This study provides novel insights into photoreceptor-RPE cell interaction in inherited blinding disorders. 
microvesicles to the extracellular space, but is degraded intracellularly. Those studies provide novel insights into protein homeostasis in the photoreceptor IS PM.

\section{Key words: blindness; extracellular vesicles; phagocytosis; retinitis pigmentosa; rhodopsin; RPE cells}

\section{Introduction}

Photoreceptor and retinal pigment epithelial (RPE) cells evolved a unique symbiotic relationship to maintain the structure and function of the photoreceptive outer segments (OSs). Each day, $\sim 5-10 \%$ of the OS is shed and engulfed by RPE cells, which digest the components-the majority of which is rhodopsin-in phagosomes located within the cytoplasm (Young, 1967; Kevany and Palczewski, 2010). This relationship is disrupted in retinal ciliopathies in which the majority of rhodopsin molecules are no longer destined to the OSs and instead mislocalize to the inner segment (IS) plasma membrane (PM; Sung et al., 1994; Li et al., 1996; Nishimura et al., 2004; Deretic et al., 2005; Adams et al., 2007; Concepcion and Chen, 2010; Hollingsworth and Gross, 2013; Nemet et al., 2015; Imanishi, 2019). In various animal models exhibiting rhodopsin mislocalization, rod photoreceptors expel rhodopsin-laden vesicles, which accumulate in the interphotoreceptor space (Li et al., 1996; Hagstrom et al., 1999; Concepcion and Chen, 2010; Lodowski et al., 2013). The interphotoreceptor space is in constant contact with RPE microvilli, which are optimally positioned for phagocytic activities (Strauss, 2005). Increasing evidence suggests that various neurons shed vesicles as means of communication and to remove unwanted materials under neurodegenerative conditions (Nagarajah, 2016; Fowler, 2019). More recently, RPE cells have been reported to take up extracellular vesicles in an in vitro cell culture model (Nicholson et al., 2020). Thus, as the first step in understanding the in vivo function of these photoreceptor-derived vesicles, we asked whether RPE cells are capable of engulfing them in a manner analogous to OS phagocytosis. Such studies will shed light on the symbiotic relationship between RPE and photoreceptors under disease states.

Received December 5, 2019; accepted April 24, 2020; First published May 6, 2020.

The authors declare no competing financial interests.

Author contributions: P.R. and Y.I. designed research; P.R. performed research; P.R. and Y.I. analyzed data; P.R. and Y.I. wrote the paper.

This work was supported by National Institutes of Health Grants R01-EY028884 (to Y.I.), R21-EY-027292 (to Y.I.), P30-EY-011373 (to Case Western Reserve University), and T32-EY-007157 (to P.R.).

Acknowledgments: We thank Dr. Sheila Baker (University of lowa, lowa City, IA) for the plasmid vector containing GFP-NKA $\alpha$, and Richard Lee [Case Western Reserve University (CWRU)] for assistance with microscopy. We also thank the CWRU Light Microscopy Imaging Core Facilities for help with confocal microscopy using a Leica TCS SP2 MP system.

Correspondence should be addressed to Yoshikazu Imanishi at yxi19@ case.edu.

https://doi.org/10.1523/ENEURO.0507-19.2020

Copyright @ 2020 Ropelewski and Imanishi

This is an open-access article distributed under the terms of the Creative Commons Attribution 4.0 International license, which permits unrestricted use, distribution and reproduction in any medium provided that the original work is properly attributed.
Unlike the degradation of OS membrane proteins, which has been relatively well-characterized (Strauss, 2005; Kevany and Palczewski, 2010), little is known about the degradation of IS PM proteins, which lack access to the RPE cells. Thus, we have initiated an effort to understand the renewal of IS PM proteins, especially focusing on class I mutant rhodopsin. In addition to the vesicle-mediated removal described above, mislocalized class I mutant rhodopsin is degraded intracellularly: once reaching the IS PM, mislocalized rhodopsin becomes internalized and subsequently degraded by lysosomes (Ropelewski and Imanishi, 2019). The IS PM component $\mathrm{Na}^{+} / \mathrm{K}^{+}$-ATPase (NKA) plays a critical role in maintaining both the dark current of photoreceptor cells (Yau and Baylor, 1989) and interactions between bipolar and photoreceptor cells (Molday et al., 2007; Friedrich et al., 2011). The lysosome-mediated removal of class I mutant rhodopsin induces co-internalization and codegradation of native NKA, compromising the structure and function of rod photoreceptors (Ropelewski and Imanishi, 2019). Another IS PM protein, HCN1 channel, plays a role in the normal electrophysiological response of photoreceptor cells, and its deficiency worsens the symptoms of retinitis pigmentosa (Schön et al., 2016). HCN1 contains a di-arginine ER retention signal that negatively regulates PM transport (Pan et al., 2015a), which is suggestive of ER-associated degradation before exiting the ER and reaching the PM. This mechanism appears to be important for regulating the expression level of HCN1 at the level of IS PM (Pan et al., 2015b). Despite improved understanding of HCN1 degradation during biosynthesis or NKA degradation under pathologic states, it is currently unknown whether and how rod photoreceptors coordinate intracellular and intercellular mechanisms for the degradation of endogenous IS PM proteins under normal physiological conditions.

In this article, we investigated the fate of microvesicles shed by rod photoreceptor cells expressing class I mutant rhodopsin. Toward that goal, we used a genetic labeling technique that allowed us to clarify the origin and destination of these secreted vesicles. Then we asked whether there is a sorting mechanism for packaging specific IS PM proteins to the microvesicles. We accomplished this by comparing IS PM-mislocalized rhodopsin and a major IS PM resident protein, NKA. To comprehensively understand the mode of renewing NKA, we compared the extracellular and intracellular mechanisms of degrading NKA. Those studies will provide novel insights into the protein homeostasis of rod photoreceptor cells both in pathologic and normal physiological conditions.

\section{Materials and Methods}

Animals. All animal procedures were approved by the Institutional Animal Care and Use Committee at Case Western Reserve University. Adult female and male frogs 
involved in transgenesis were purchased from Nasco and housed at $16^{\circ} \mathrm{C}$ under a $12 \mathrm{~h} \mathrm{light/dark} \mathrm{cycle.} \mathrm{All} \mathrm{tadpoles}$ used for experiments were housed at $16^{\circ} \mathrm{C}$ in $24 \mathrm{~h}$ of darkness. Tadpoles were fed spirulina (https://nuts.com). Both male and female tadpoles were used for all experiments.

Reagents. Unless otherwise specified, all reagents were purchased from either Thermo Fisher Scientific or SigmaAldrich.

Molecular cloning. DNA expression vectors were generated by standard methods combining PCR, DNA recombination, and site-directed mutagenesis. The expression vector containing the Xenopus opsin promotor (XOP) followed by GFP-NKA $\alpha 3$ (Laird et al., 2015) was modified. The vector contained an Agel site upstream of GFP-NKA $\alpha$. Site-directed mutagenesis was used to create an additional Agel site after the region encoding GFP; the GFP coding region was then removed using Agel enzyme and replaced with cDNA encoding Dendra2 fluorescent protein. The resulting DNA fragment was introduced downstream of XOP and upstream of NKA $\alpha$ using In-Fusion Cloning Kit (TaKaRa). The plasmid vectors containing XOP-Rho-Dend2-1D4 and XOP-Rho ${ }_{\mathrm{Q} 344 \text { ter- }}{ }^{-}$ Dend2 were previously generated (Lodowski et al., 2013). All the vectors contained polyadenylation signals following the coding and noncoding regions.

Transgenesis of Xenopus laevis. Transgenic Xenopus laevis were produced using the intracytoplasmic sperm injection method following the previously published procedure and screened for the presence of Dendra2 fluorescence (Sparrow et al., 2000; Smith et al., 2006; Lodowski et al., 2013). In order to prevent photobleaching, light-dependent photoreceptor degeneration, and unintended photoconversion of fluorescent protein Dendra2, tadpoles were reared in $24 \mathrm{~h}$ darkness.

Immunohistochemistry. $X$. laevis retinal sections $(12 \mu \mathrm{m})$ were prepared and stored at $-80^{\circ} \mathrm{C}$, as described previously (Lodowski et al., 2013; Ropelewski and Imanishi, 2019). Slides were removed from $-80^{\circ} \mathrm{C}$ storage conditions and warmed using a $37^{\circ} \mathrm{C}$ incubator (CCC $0.5 \mathrm{~d}$, Boekel Scientific). After thawing the sections, all of the following procedures were conducted at room temperature. Individual sections were encircled by an ImmEdge Pen (Vector Laboratories). Sections were then rehydrated and blocked in a $1.5 \%$ normal goat serum diluted in PBS solution $\left(137 \mathrm{~mm} \mathrm{NaCl}, 2.7 \mathrm{~mm} \mathrm{KCl}, 8 \mathrm{~mm} \mathrm{Na} \mathrm{HPO}_{4}, 2 \mathrm{~mm}\right.$ $\mathrm{KH}_{2} \mathrm{PO}_{4}$ ) for 30 min. Blocking solution was then removed, and primary antibody mouse anti-NKA $\alpha$ (1:100; M7-PBEIX, Santa Cruz Biotechnology) diluted in PBS containing $0.1 \%$ Triton X-100 (PBST) was added; sections were incubated for $\sim 14 \mathrm{~h}$ and then washed three times with PBST for $10 \mathrm{~min}$ each. After the washes, sections were incubated in PBST containing phalloidin conjugated with Alexa Fluor 633 (1:100; catalog \#A22284, Thermo Fisher Scientific), Hoechst 33342 dye (1:500), and goat anti-mouse IgG conjugated either with Alexa Fluor 488 (1:100; catalog \#115545-166, Jackson ImmunoResearch) or with Cy3 (1:100; catalog \#115-165-166, Jackson ImmunoResearch) for $1 \mathrm{~h}$ followed by three additional $10 \mathrm{~min}$ washes with PBST. After these washes, individual sections were mounted with a coverslip and one drop of VECTASHIELD mounting medium (Vector Laboratories). Nail polish (Electron
Microscopy Sciences) was used to seal the coverslip, and the sections were dried for $>30$ min before imaging.

Preparation of retina explant for confocal imaging. Tadpoles were killed, decapitated, and their eyes were removed as described previously (Ropelewski and Imanishi, 2019). The RPE layers were separated from the retinas, which were immediately placed into a glass-bottom dish (catalog \#P35G-1.5-14-C, MatTek) containing modified Wolf medium (D-glucose, $700 \mathrm{mg} / \mathrm{L}, 30 \mathrm{~mm} \mathrm{NaHCO} 3,55 \%$ MEM, 31\% sodium-free BBS, 10\% FBS; Lodowski et al., 2013). Equilibration of Wolf medium was achieved by incubating the dish containing the medium in an incubation chamber (Tokai Hit) supplied with $5 \% \mathrm{CO}_{2}$ and $95 \% \mathrm{O}_{2}$ (Airgas; Nemet et al., 2014). The glass-bottom dishes were coated with Cell-Tak Cell and Tissue Adhesive (Corning). A circular glass coverslip (12 mm diameter; $0.13-0.17 \mathrm{~mm}$ thickness) was used to seal and flatten the retinas before imaging.

Confocal microscopy, image analysis, quantification, and statistical analysis. All images were acquired using a Leica TCS SP2 laser-scanning confocal/multiphoton microscope system equipped with the following four lasers for excitation: $488 \mathrm{~nm}$ argon ion, $543 \mathrm{~nm} \mathrm{HeNe}, 633 \mathrm{~nm} \mathrm{HeNe}$, and tunable Chameleon XR Ti:Sapphire laser (Leica Microsystems), as described previously (Ropelewski and Imanishi, 2019). An HCX PL APO CS $40.0 \times 1.25$ oil UV objective lens was used for imaging. Diameters of vesicular structures were measured using the ImageJ line function tool (Schneider et al., 2012). For irregularly shaped vesicles, which were approximated as ovals, the diameter was calculated by drawing a line at a $45^{\circ}$ angle from the cross section to focal points on the edges of the structure. This calculated distance between the focal points was used as the approximated diameter. For the quantitative studies involving drug treatments, the same laser power and imaging condition were used for all the samples. The cytoplasmic space was highlighted manually, and its mean fluorescence intensity measured using the ImageJ freehand selection tool (Schneider et al., 2012). The background noise was determined from blank space where no cells were observed and was subtracted from the measurements. For quantification of cytoplasmic Dend2-NKA $\alpha$, statistical outliers were identified by the interquartile range method and removed (Kokoska and Zwillinger, 2000). An estimation plot graph was generated via Prism software version 8.4.0 (GraphPad Software). In retina explants from $9 \mathrm{~d}$ postfertilization (DPF) animals, we quantified the number of vesicles associated with individual rod cells. For this purpose, vesicles within $2 \mu \mathrm{m}$ of each rod photoreceptor cell surface were considered to be associated with the cell. Each vesicle was considered to be associated with only one cell. Unless otherwise noted, the quantitative data are represented as the mean \pm SD ( $n=4$ animals or structures thereof). In comparing two populations, $* p<0.001$ by Student's $t$ test was considered statistically significant.

\section{Results}

\section{IS-mislocalized rhodopsin is secreted and uptaken by RPE cells}

Based on past studies (Li et al., 1996; Concepcion and Chen, 2010; Lodowski et al., 2013), class I mutant 
A

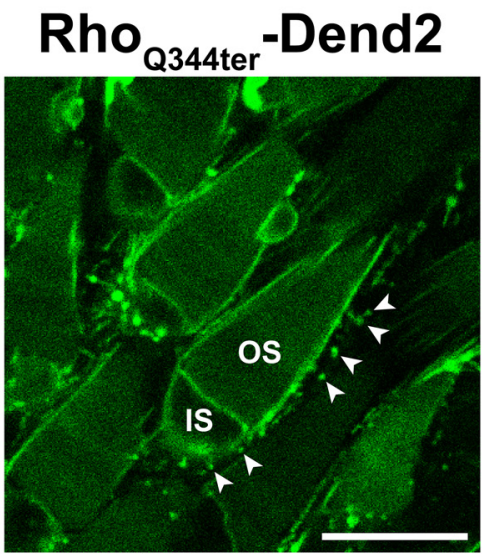

C

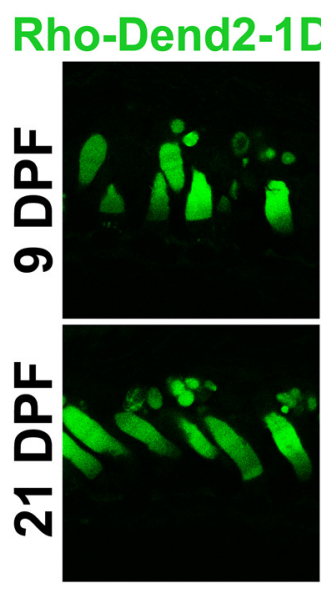

\section{B Rho ${ }_{\text {Q344ter }}$}
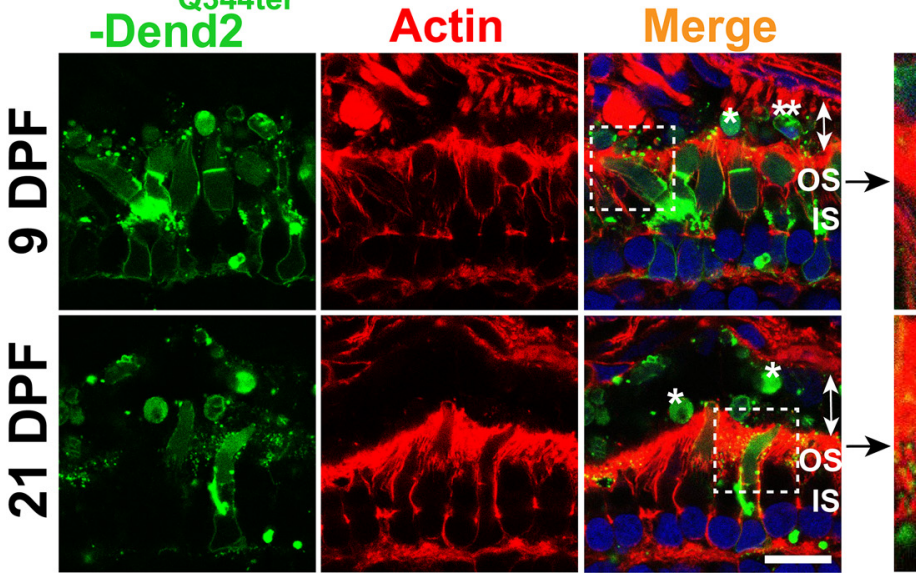

Zoom

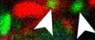

$4 \lambda$. 44

1

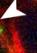
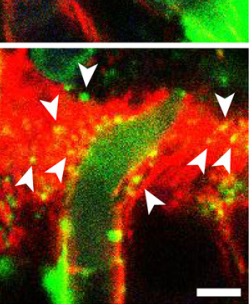

D
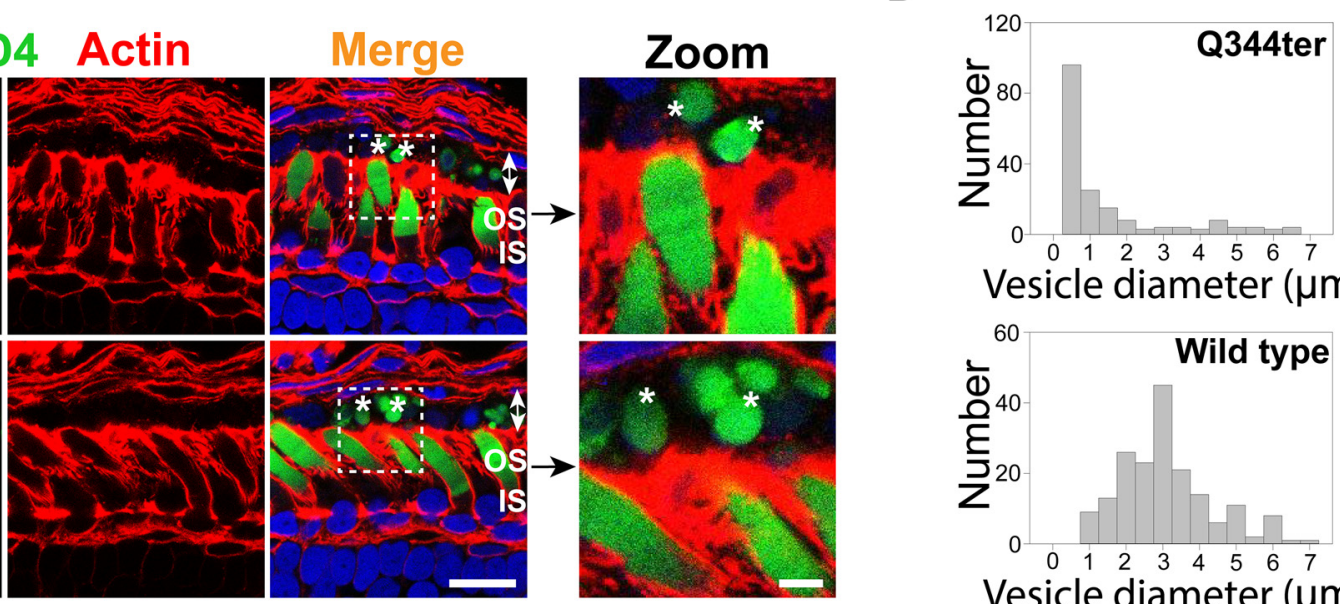

Vesicle diameter ( $\mu \mathrm{m})$

Figure 1. Class I mutant rhodopsin is released as microvesicles before being engulfed by RPE cells. $\boldsymbol{A}$, Live retina explant was imaged at 9 DPF. Vesicles (arrowheads) were observed in the extracellular space surrounding rods expressing class I mutant rhodopsin, Rho ${ }_{Q 344 t e r}$-Dend2. B, Cross sections of retinas expressing Rho ${ }_{\mathrm{Q} 344 t e r}$-Dend2 (Dend2, green) labeled with phalloidin-Alexa Fluor

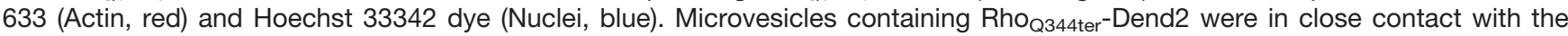
actin filaments of the RPE microvilli (Zoom, arrowheads) and within RPE cytoplasmic space (Merge, indicated by double-headed arrows). Microvesicles were observed both at 9 DPF (top panels) and 21 DPF (bottom panels). Large OS fragments (single asterisk) or cell bodies (double asterisks) also existed within the RPE cell layer (double-headed arrows). $\boldsymbol{C}$, Cross sections of retinas expressing Rho-Dend2-1D4 (Dend2, green) were labeled with phalloidin-Alexa Fluor 633 (Actin, red) and Hoechst 33342 dye (Nuclei, blue). OS fragments (Zoom, single asterisk) containing Rho-Dend2-1D4 were visible in cytoplasmic space (Merge, double-headed arrows) of the RPE cells. Retinas were imaged either at 9 DPF (top panels) or at 21 DPF (bottom panels). $\boldsymbol{D}$, Size distribution of green fluorescent structures/vesicles found in the RPE of animals expressing Rho ${ }_{\mathrm{Q} 344 t e r}$-Dend2 (Q344ter, based on 180 structures from $n=4$ animals) or Rho-Dend2-1D4 (wild type, based on 180 structures from $n=4$ animals) at 21 DPF. Scale bars: Zoom, $1 \mu \mathrm{m}$; other panels, $10 \mu \mathrm{m}$.

rhodopsin molecules are secreted as vesicles that accumulate in the interphotoreceptor space. We reproduced these findings in a $X$. laevis model that expresses class I mutant (Q344ter) rhodopsin fused to Dendra2 in rod photoreceptor cells. Rhodopsin-laden vesicles were released into the extracellular space of retina explant culture (Fig. 1A, arrowheads). At $9 \mathrm{DPF}$, the majority ( $\sim 93 \%)$ of rods expressing $\mathrm{Rho}_{\mathrm{Q} 344 t e r}$-Dend2 were associated with vesicles $(3.45 \pm 0.81$ vesicles associated with a single rod, $n=160$ rod cells from four animals) in the extracellular space. As apical extensions of RPE cells are involved in phagocytic activities in the interphotoreceptor space, we asked whether RPE cells are capable of engulfing and clearing these vesicles. Apical microvilli and basal plasma membrane of RPE cells are densely coordinated with F-actins (Bonilha et al., 1999), which were labeled with fluorescently conjugated phalloidin (Fig. 1B,C, actin). Cytoplasmic portions of the RPE cells were barely labeled and readily recognizable from apical and basolateral borders (Fig. 1B,C, merge, double-headed arrows). In retinas expressing $\mathrm{Rho}_{\mathrm{Q} 344 t e r}$-Dend2, microvesicles were associated with the RPE microvilli (Fig. 1B, zoom, arrowheads), indicating these vesicles were in the process of being engulfed.

We obtained two lines of evidence that a significant fraction of microvesicles released by $\mathrm{Rho}_{\mathrm{Q} 344 \text { ter-Dend2- }}$ 


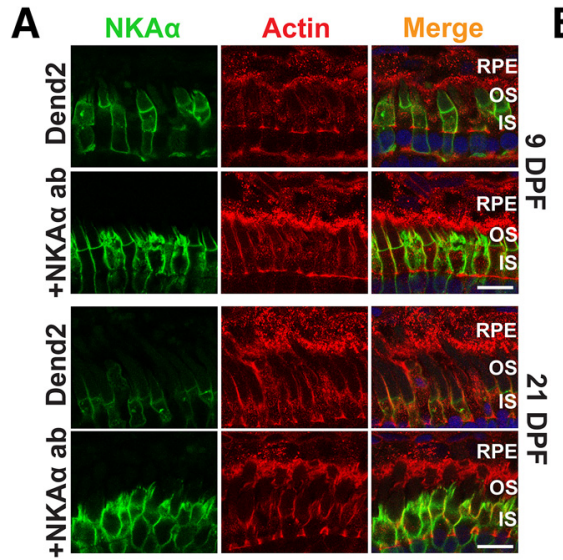

B

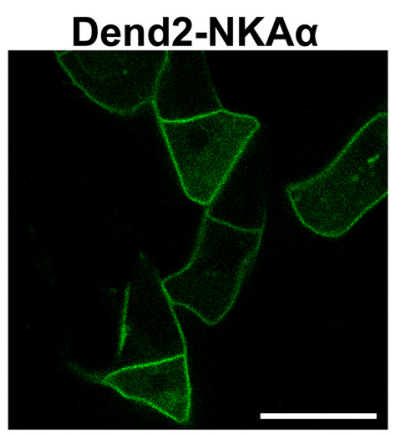

Figure 2. NKA $\alpha$ is not released in extracellular vesicles under normal physiological conditions. $\boldsymbol{A}$, Cross sections of retinas expressing Dend2-NKA $\alpha$ were labeled with phalloidin-Alexa Fluor 633 (Actin, red) and Hoechst 33342 dye (Nuclei, blue). Retinas were 9 DPF (top two rows) or 21 DPF (bottom two rows). For each panel, either Dend2-NKA $\alpha$ fluorescence was imaged directly (Dend2, green) or NKA $\alpha$ (both endogenous and Dend2-NKA $\alpha$ ) was visualized by immunofluorescence (+NKA $\alpha$ ab, green). B, Live retina explant was imaged at 9 DPF. NKA $\alpha$-containing extracellular vesicles were not observed in the vicinity of rod cells expressing Dend2NKA $\alpha$. Scale bars, $10 \mu \mathrm{m}$.

positive rods are engulfed by RPE cells. First, $\sim 40 \%$ of all vesicular structures in the cytoplasm of the RPE (Fig. $1 B, D$, Q344ter) had diameters within the 95\% confidence interval $(0.34-0.38 \mu \mathrm{m})$ of the size distribution demonstrated by extracellular microvesicles (Fig. 1A). Second, because $\mathrm{RhO}_{\mathrm{Q} 344 \mathrm{ter}}$-Dend2 is synthesized in rods under the regulation of the Xenopus rhodopsin promoter, these $\mathrm{Rho}_{\mathrm{Q} 344 t e r}$-Dend2-containing microvesicles within the RPE must have originated from rod cells. Thus, under pathologic conditions, RPE cells are capable of engulfing rhodopsin-laden microvesicles that were secreted into the interphotoreceptor space.

Under normal conditions, wild-type rhodopsin is known to be shed in OS membrane fragments, which are phagocytosed by RPE cells. Consistently, wild-type rhodopsin fused to Dendra2 (Rho-Dend2-1D4) was observed in phagosomes within RPE cells (Fig. 1C, asterisks). Structures containing Rho-Dend2-1D4 were significantly larger (2.89 $\pm 1.31 \mu \mathrm{m}, n=180$ structures) than the extracellular vesicles released by rods expressing $\mathrm{Rho}_{\mathrm{Q} 344 \mathrm{ter}}{ }^{-}$ Dend2 in retina explant culture (Fig. 1A, arrowheads; $0.36 \pm 0.14 \mu \mathrm{m}, n=180$ vesicles, $p<0.001)$. While the majority of Rho ${ }_{\mathrm{Q} 344 \mathrm{ter}}$-Dend2 mislocalizes to the IS PM of rod cells, a small fraction still reaches the OS, likely due to cotrafficking with endogenous rhodopsin (Concepcion and Chen, 2010). Thus, larger OS fragments exhibiting Dendra2 fluorescence were also observed in the RPE cytoplasm of animals expressing Rho ${ }_{\mathrm{Q} 344 t e r}$-Dend2 (Fig. 1B, merge, asterisks). We also observed large cellular fragments that are likely derived from rod photoreceptor inner segments or cell bodies (Fig. $1 B$, merge, double asterisks). Some of those fragments contained DNA and were surrounded by $\mathrm{Rho}_{\mathrm{Q} 344 t e r}$-Dend2-positive membranes, suggesting that they were rod photoreceptor cell bodies. Unlike the RPE cells of animals expressing $\mathrm{Rho}_{\mathrm{Q} 344 t \mathrm{ter}^{-}}$ Dend2, in which $40 \%$ of vesicular structures are at the size of microvesicles, nearly all the structures in the RPE layer of animals expressing Rho-Dend2-1D4 animals were larger than microvesicles (Fig. 1D, compare Q344ter to wild type), confirming that wild-type rhodopsin is shed and phagocytosed as large OS fragments but not as microvesicles.

\section{RPE cells do not contribute to the degradation of NKA under normal physiological conditions}

The above experiments suggested that IS PM mislocalized rhodopsin is packaged in microvesicles that are engulfed by RPE cells. We asked whether a similar mechanism removes IS PM resident proteins. NKA is highly expressed on the photoreceptor IS PM (Kwok et al., 2008) and is essential for maintaining the dark current (Schneider and Kraig, 1990). Thus, we focused on the degradation of the $\alpha 3$-subunit of NKA (NKA $\alpha$ ), which is enriched in photoreceptor neurons (Wetzel et al., 1999). To emulate the above experiments visualizing class I mutant rhodopsin, the NKA $\alpha 3$-subunit was fused to Dendra2 (Dend2-NKA $\alpha$ ) and expressed in rod photoreceptors under the regulation of Xenopus rhodopsin promoter. To visualize the borders of RPE cells, retinas expressing Dend2-NKA $\alpha$ were stained with fluorescently conjugated phalloidin (Fig. 2A, actin). In these retinas, Dend2-NKA $\alpha$ was not observed in the RPE cells (Fig. 2A, Dend2, RPE), indicating that the photoreceptors were not secreting Dend2-NKA $\alpha$. Consistently, Dend2-NKA $\alpha$ was not released to the extracellular space as vesicles in live retina explant culture (Fig. 2B). To exclude the possibility that vesicles were present but not visible due to our limited detection sensitivities of Dendra2, we enhanced the fluorescence signal by labeling these samples with antiNKA $\alpha$ (Fig. $2 A,+$ NKA $\alpha$ ab). Regardless of rigorous labeling techniques, no vesicles were observed in the RPE layer. We obtained similar results with wild-type (nontransgenic) animals, which did not express Dend2-NKA $\alpha$. In these animals, endogenous NKA $\alpha$, labeled by immunofluorescence, was not observed in microvesicles extracellularly or within RPE cells (Fig. $3 A$, nontransgenic), confirming that RPE cells do not contribute to the 


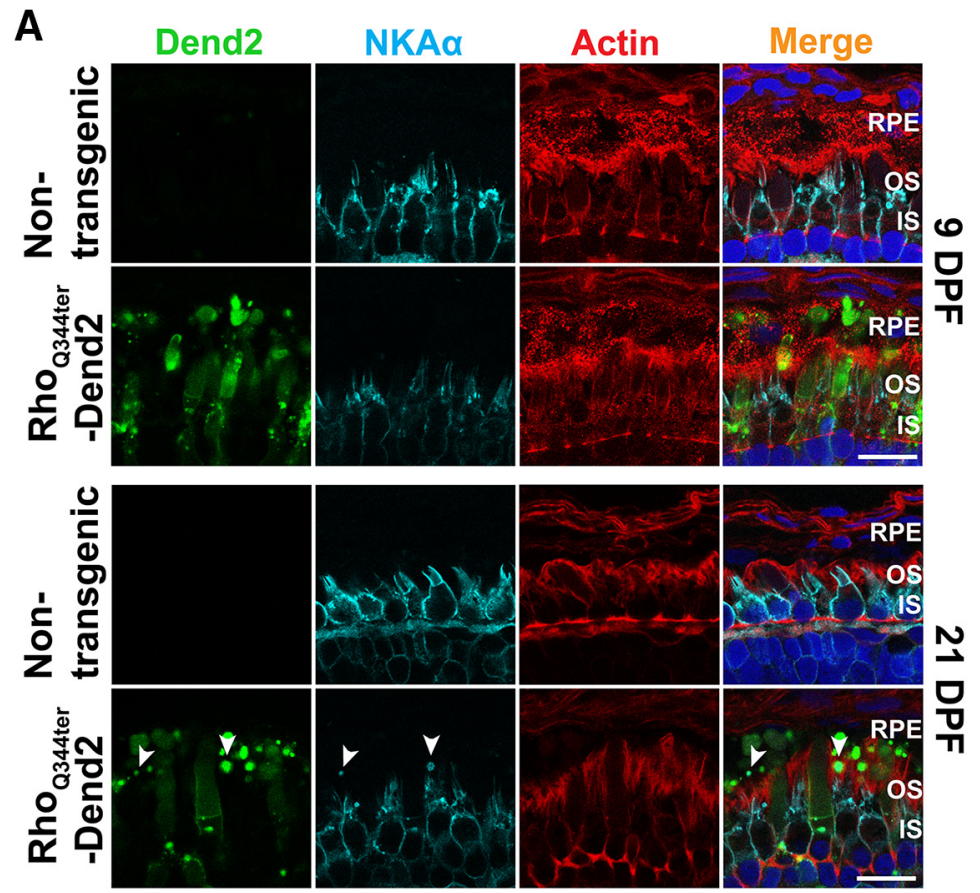

B

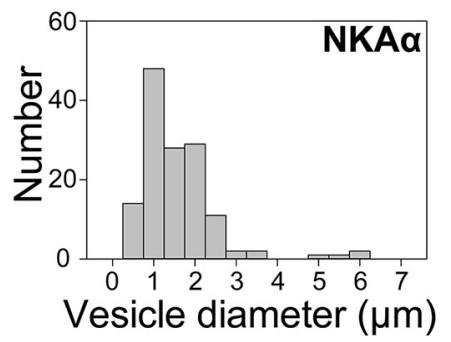

c

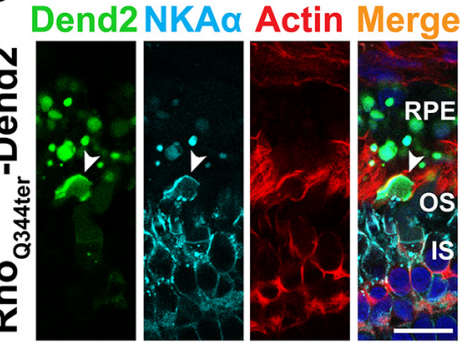

Figure 3. IS fragments containing NKA $\alpha$ are phagocytosed by RPE at a late stage of photoreceptor degeneration. $\boldsymbol{A}$, Cross sections of retinas that express either no transgenic protein (Nontransgenic) or class I mutant rhodopsin fused to Dendra2 (Rho ${ }_{\mathrm{Q} 344 t e r}{ }^{-}$ Dend2). Retinas were 9 DPF (top two rows) or 21 DPF (bottom two rows). Sections were labeled with anti-NKA $\alpha$ antibody (NKA $\alpha$, cyan), phalloidin-Alexa Fluor 633 (Actin, red), and Hoechst 33342 dye (Nuclei, blue). Photoreceptor-derived NKA $\alpha$ was not shed in microvesicles at either 9 or 21 DPF, whereas large vesicular structures (arrowheads) containing NKA $\alpha$ were occasionally seen in contact with RPE microvilli or inside RPE layer at 21 DPF. B, Histogram indicating the size distribution of structures containing $\mathrm{NKA} \alpha$ within the RPE layer. Retinas expressing Rho ${ }_{\mathrm{Q} 344 t e r}$-Dend2 were analyzed at $21 \mathrm{DPF}$. $\boldsymbol{C}, \mathrm{A}$ cross section of $21 \mathrm{DPF}$ retina expressing $\mathrm{Rho}_{\mathrm{Q} 344 \mathrm{ter}}$-Dend2 and labeled with anti-NKA $\alpha$ (NKA $\alpha$, cyan), phalloidin-Alexa Fluor 633 (Actin, red) and Hoechst 33342 dye (Nuclei, blue). In this view, a large inner segment fragment containing NKA $\alpha$ (arrowhead) is being engulfed by an RPE cell. Scale bars, $10 \mu \mathrm{m}$.

degradation of endogenous NKA $\alpha$ under normal physiological conditions.

\section{NKA $\alpha$ is not cosecreted along with class I mutant rhodopsin as microvesicles}

In rods expressing class I mutant rhodopsin $\left(\mathrm{Rho}_{\mathrm{Q} 344 \mathrm{ter}}{ }^{-}\right.$ Dend2), NKA $\alpha$ is downregulated because of its codegradation with $\mathrm{Rho}_{\mathrm{Q} 344 \mathrm{ter}}$-Dend2 by lysosomes (Ropelewski and Imanishi, 2019). As our current study demonstrates engulfment of vesicles containing $\mathrm{RhO}_{\mathrm{Q} 344 \mathrm{ter}}$-Dend2 by RPE cells, we asked whether NKA $\alpha$ was also co-degraded by this route. In rods expressing $\mathrm{RhO}_{\mathrm{Q} 344 t e r}$-Dend2, active degradation of $\mathrm{NKA} \alpha$ proteins was observed around 9 DPF (Ropelewski and Imanishi, 2019). At this early stage (9 DPF), Rho ${ }_{Q 344 t e r}$-Dend2 is actively released as vesicles from rods (Fig. $1 A$ ) and engulfed by RPE cells (Fig. 1B). We used anti-NKA $\alpha$ antibody to label endogenous $\mathrm{NKA} \alpha$ in retinas expressing Rho $_{\text {Q344ter-Dend2 }}$ at 9 and 21 DPF (Fig. 3A, Rho ${ }_{\text {Q344ter }}$ Dend2, NKA $\alpha$, cyan). NKA $\alpha$ was not observed in Rho $_{\mathrm{Q} 344 t e r}$-Dend2-positive vesicles inside the RPE cells at 9 DPF (Fig. 3A, Rho ${ }_{Q 344 t e r}$-Dend2, RPE). Thus, NKA $\alpha$ is not secreted together with $\mathrm{Rho}_{\mathrm{Q} 344 \mathrm{ter}}$-Dend2 as microvesicles.

In later stage (21 DPF) animals expressing $\mathrm{Rho}_{\mathrm{Q} 344 t \mathrm{ter}^{-}}$ Dend2 (Fig. 3A, $21 \mathrm{DPF}, \mathrm{RhO}_{\mathrm{Q} 344 \mathrm{ter}}$-Dend2), photoreceptor degeneration advances (Lodowski et al., 2013). Likely because of rod degeneration, we observed fewer vesicles $(* p<0.001)$ in the retinas of older (21 DPF) animals $(16.7 \pm 3.3$ vesicles $/ 100 \mu \mathrm{m}$ retina length) than in younger (9 DPF) animals ( $33.9 \pm 5.6$ vesicles $/ 100 \mu \mathrm{m}$ retina length). Coincidentally with the degenerative events, NKA $\alpha$ 
proteins originating from IS PM were occasionally observed in structures in contact with RPE microvilli or within the cytoplasm of the RPE (Fig. 3A, 21 DPF, arrowheads). These structures contained $\mathrm{Rho}_{\mathrm{Q} 344 \mathrm{ter}^{-}}$ Dend2, indicating they originated from rods experiencing rhodopsin mislocalization. The size of these structures $(1.33 \pm 0.94 \mu \mathrm{m}$, based on 138 structures; Fig. $3 B)$ was significantly $(* p<0.001)$ larger than that of the extracellular microvesicles containing $\mathrm{Rho}_{\mathrm{Q} 344 t e r}$-Dend2 $(0.36 \pm 0.14 \mu \mathrm{m})$ in retina explant culture (Fig. 1A). Occasionally, large NKA $\alpha$-containing fragments comparable in size to a rod IS were seen in the process of being engulfed by RPE cells (Fig. $3 C$, arrowhead). Thus, the NKA $\alpha$-containing structures observed at 21 DPF are likely cellular debris from ISs that degenerated as a result of $\mathrm{RhO}_{\mathrm{Q} 344 t e r}$-Dend2 expression. In retinas not expressing $\mathrm{Rho}_{\mathrm{Q} 344 \mathrm{ter}}$-Dend2, we did not observe photoreceptor-derived NKA $\alpha$ in RPE cells either at 9 or 21 DPF (Fig. $2 A, 9$ DPF and 21 DPF). In summary, RPE cells are capable of engulfing cellular debris containing IS PM protein NKA $\alpha$ as a result of photoreceptor degeneration, but NKA $\alpha$ itself is not cosecreted with class I mutant rhodopsin as microvesicles.

\section{NKA $\alpha$ is potentially degraded within rod photoreceptors}

IS PM protein NKA $\alpha$ is not removed via vesicle release and absorption by the RPE cells under normal physiological conditions. Therefore, we explored whether intracellular mechanisms contribute to the degradation of NKA $\alpha$. Two potential contributors are lysosomes and proteasomes. To test the in vivo degradation of $\operatorname{NKA} \alpha$, we treated animals expressing Dend2-NKA $\alpha$ with lysosome inhibitor bafilomycin A1 (BA1) and/or proteasome inhibitor bortezomib (Bort) for various lengths of time (Fig. $4 A$ ). As controls, animals were treated only with the vehicle (DMSO) for $24 \mathrm{~h}$ (Fig. 4A, control). BA1 (100 nm) did not significantly increase the amount of intracellular Dend2NKA $\alpha$ (Fig. $4 B ; p>0.5$ for 24-144 h BA1-treated vs control), suggesting that lysosomes do not contribute to the NKA $\alpha$ degradation. Bort (100 nm) increased the amount of Dend2-NKA $\alpha$ in the IS cytoplasm significantly after $24 \mathrm{~h}$ (37.0\% increase) and $72 \mathrm{~h}$ (36.1\% increase) of treatment (Fig. 4B; $p$ values $<0.001$ for $24-72 \mathrm{~h}$ Bort-treated vs control). Similar accumulations were observed when animals received combined treatment of BA1 and Bort (Combo) for $24 \mathrm{~h}$ (49.6\% increase) or $72 \mathrm{~h}(61.6 \%$ increase; Fig. $4 B ; p$ values $<0.001$ for $24-72$ h Combo vs control). We noted that the amount of intracellular Dend2NKA $\alpha$ decreased after 144 h of Bort treatment $(p<0.002$, $72 \mathrm{~h}$ Bort vs $144 \mathrm{~h}$ Bort and $72 \mathrm{~h}$ Combo vs $144 \mathrm{~h}$ Combo). A potential reason for this decrease is resistance to Bort after long-term treatment, as characterized previously (Rückrich et al., 2009; Wu et al., 2016); this adaptive mechanism leads to an increase in proteasome subunit transcription, thus partially overcoming Bort inhibition. Therefore, we conducted an additional experiment using marizomib (Mariz), which was demonstrated to inhibit proteolytic activity even in Bort-adapted cells (Merin and Kelly, 2014). As anticipated, an increase in intracellular
A

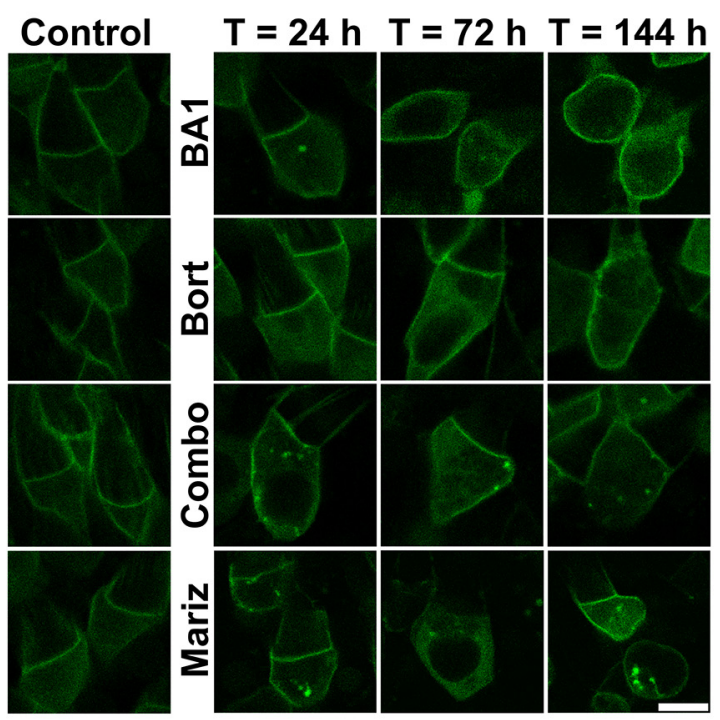

B

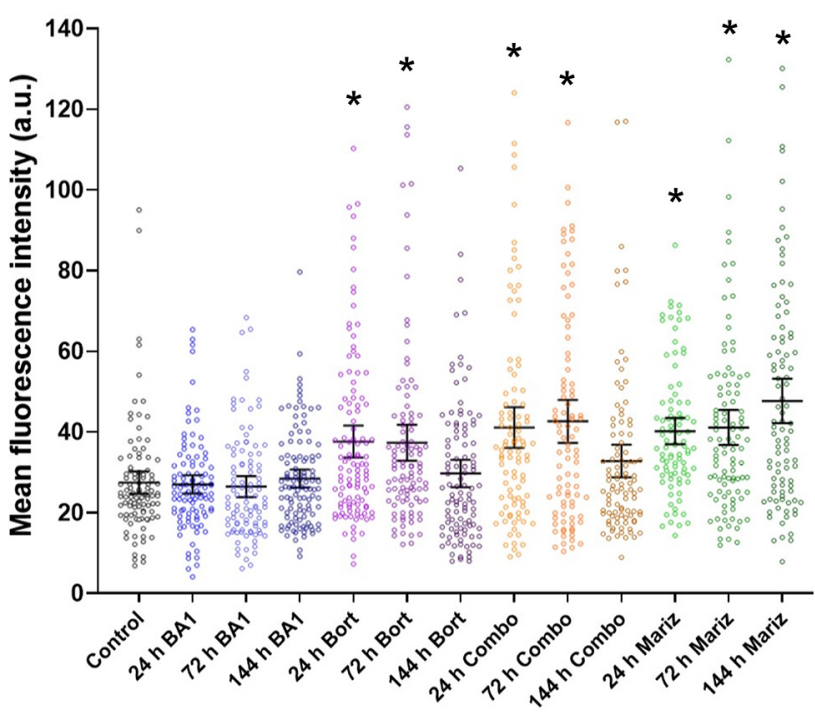

Figure 4. NKA $\alpha$ proteins are degraded intracellularly in rod photoreceptors. $\boldsymbol{A}$, Representative images of rod cells from animals expressing Dend2-NKA $\alpha$ and treated with either the vehicle (DMSO, control), 100 nм BA1, 100 nм Bort, a combination of both BA1 and Bort (Combo), or 250 nm Mariz for 24-144 h. Scale bar, $5 \mu \mathrm{m}$. B, Estimation plot analysis of Dend2-NKA $\alpha$ concentration. Average fluorescence intensities of Dend2-NKA $\alpha$ were measured in the cytoplasmic region of individual rods (each dot). The error bars represent the mean \pm 1.96 SEs $(95 \%$ confidence interval). The average fluorescence intensities are indicated on the $y$-axis [arbitrary unit (a.u.)]. $* p<0.001$ by Student's $t$ test (each treatment condition vs control). At least 92 rod cells $(n=92)$ from four independent animals were subjected to quantification for each condition.

Dend2-NKA $\alpha$ was observed after $24 \mathrm{~h}$ (46.4\% increase), $72 \mathrm{~h}(49.7 \%$ increase), and $144 \mathrm{~h}(73.7 \%$ increase $)$ of Mariz (250 nм) treatment (Fig. 4B; $p$ values $<0.001$ for 24-144 h Mariz vs control). Considering that proteasome inhibition resulted in the accumulation of otherwise degraded Dend2-NKA $\alpha$ proteins, the proteasome is likely 
involved in the degradation of NKA $\alpha$ proteins. In summary, further studies are necessary to corroborate these findings because of the small effect of proteasome inhibition on protein accumulation.

\section{Discussion}

In this article, we demonstrated that microvesicles secreted by rod photoreceptors are engulfed by and incorporated into RPE cells. These microvesicles are distinct from shed rod outer segment fragments phagocytosed by RPE cells. Under pathologic conditions such as those observed for rods expressing class I mutant rhodopsin, rhodopsin-laden microvesicles are actively shed into the interphotoreceptor space (Li et al., 1996; Concepcion and Chen, 2010; Lodowski et al., 2013). The sizes of the vesicles (95\% confidence interval, $0.34-0.38 \mu \mathrm{m})$ in our $X$. laevis model were comparable to the sizes of vesicles $(0.1-0.2 \mu \mathrm{m})$ in a murine model of rhodopsin class I mutation (Concepcion and Chen, 2010), indicating that the shedding of rhodopsin-laden vesicles is a conserved mechanism to expel mislocalized rhodopsin from rod photoreceptors. The past electron microscopy studies indicated that rhodopsin-laden vesicles are budding off of the IS PM in different animal models of rhodopsin mislocalization (Blanks and Spee, 1992; Hagstrom et al., 1999; Kondo et al., 2009). Previously (Lodowski et al., 2013), we used the photoconversion technique to address where microvesicles originated from in rod photoreceptor cells. In these experiments, we initially photoconverted the entire population of $\mathrm{RhO}_{\mathrm{Q} 344 \text { ter }}$-Dend2 into red, and followed the new synthesis and trafficking of green $\mathrm{RhO}_{\mathrm{Q} 344 \text { ter }^{-}}$ Dend2 in the cells. These experiments revealed that microvesicles, $2 \mathrm{~d}$ after photoconversion, were yellow in color, demonstrating a mixture of red and green isoforms of Rho ${ }_{\mathrm{Q} 344 t e r}$-Dend2. During this time frame, the IS PM had a similar composition of green and red $\mathrm{Rho}_{\mathrm{Q} 344 \text { ter }^{-}}$ Dend2 as the microvesicles. We believe that the OS is not a source of microvesicles because such a mixture of old (red) and newly synthesized (green) protein does not occur in the OS compartment. In the OS compartment, new proteins are compartmentalized in new disks near the basal region of OSs, and old proteins are compartmentalized in the distal portion of the OSs. Six days after photoconversion, rod photoreceptors synthesized more green $\mathrm{RhO}_{\mathrm{Q} 344 \mathrm{ter}}$-Dend2, which was mistrafficked to the IS PM, resulting in higher green fluorescence in this compartment. Coincidentally, the microvesicles observed $6 \mathrm{~d}$ after photoconversion contained a higher amount of new (green) Rho ${ }_{\mathrm{Q} 344 t e r}-$ Dend2. Therefore, the color composition of $\mathrm{RhO}_{\mathrm{Q} 344 \text { ter- }}$ Dend2 proteins on the IS PM is strongly correlated that of the microvesicles, indicating that the microvesicles are originating from the IS PM.

The past studies indicate that mouse models of ciliopathies caused by Tulp1 and IFT88 mutations manifest rhodopsin mislocalization and accumulation of rhodopsinladen vesicles, as was observed in this study (Hagstrom et al., 1999; Pazour et al., 2002). Those proteins are considered essential for cilia-associated signaling and intraflagellar transport (Hagstrom et al., 2001; Nemet et al., 2015). In the Tulp1-deficient murine model, the number of rhodopsin-laden vesicles peaks at early stages (17-21 d old) when photoreceptors are still maturing (Hagstrom et al., 2001). Consistently, there were more vesicles in the early stage (9 DPF) than in the later stage (21 DPF) in our Xenopus model. Anatomically, the interphotoreceptor space where these vesicles are shed is accessible by RPE apical membrane extensions that are optimal for phagocytic activities. To clarify the source of the microvesicles, we fluorescently labeled the microvesicles with Rho ${ }_{\mathrm{Q} 344 t e r}$-Dend2. Because Rho ${ }_{\mathrm{Q} 344 t e r}-$ Dend2 was specifically expressed in rods under the regulation of rhodopsin promoter, we concluded that fluorescent vesicles observed in RPE originated from rod photoreceptors. Engulfment of these vesicles is potentially neuroprotective as it removes rhodopsin molecules that cannot productively engage in phototransduction (Alfinito and Townes-Anderson, 2002), prevents overaccumulation of vesicular structures in the interphotoreceptor space, and potentially allows biomolecules to be recycled.

Those microvesicles did not contain NKA $\alpha$, which is the major component of the photoreceptor IS PM. Thus, removal of class I mutant rhodopsin through this route will not result in the downregulation of NKA $\alpha$. This is in contrast to the intracellular route of rhodopsin removal, which coincidentally downregulates NKA $\alpha$ (Ropelewski and Imanishi, 2019). In this route, class I mutant rhodopsin mislocalized to the IS PM is internalized together with NKA $\alpha$ to intracellular lysosomes, where they are codegraded, causing photoreceptor dysfunction (Ropelewski and Imanishi, 2019). To compare this behavior of class I mutant with another IS PM protein under the same condition, we expressed Dend2-NKA $\alpha$ in rods under the Xenopus rhodopsin promoter. Despite using the same expression system, we did not observe Dend2-NKA $\alpha$ secreted in microvesicles. Therefore, microvesicle secretion does not appear to be a normal route of degradation for IS PM-resident protein. Packaging of mislocalized rhodopsin into microvesicles is a unique cellular response to the expression of class I mutant rhodopsin, and there are specific sorting mechanisms that differentiate class I mutant rhodopsin and NKA.

To study the behavior of endogenous $\operatorname{NKA} \alpha$, we used anti-NKA $\alpha$ antibody, which specifically labeled NKA $\alpha$ in the photoreceptor IS PM layer but did not label the NKA $\alpha$ proteins that are localized to the apical membrane of RPE cells (Hu and Bok, 2001). Under conditions of rhodopsin mislocalization, we did not observe NKA $\alpha$ in the RPE layer of tadpoles at $9 \mathrm{DPF}$, when photoreceptor degeneration is not prominent and microvesicles are actively released. In later stages (21 DPF) when photoreceptor degeneration becomes apparent, we started observing structures containing NKA $\alpha$ around the microvilli and within the RPE cells. We also captured a large NKA-containing IS fragment in the process being engulfed by RPE cells. In line with this notion, IS components are phagocytosed by the RPE cells during photoreceptor degeneration caused by class II mutant rhodopsin (Sakami et al., 2019). Thus, these experiments confirm that endogenous NKA $\alpha$ proteins are not secreted as microvesicles under physiological or pathophysiological conditions. Considering that microvesicles are not removing NKA $\alpha$ 
from rods, we explored intracellular degradation pathways within rods. Using pharmacological approaches, we found that NKA $\alpha$ is likely degraded by proteasomes, but not by lysosomes, within rods. This is in line with our previous study in which PSmOrange-NKA $\alpha$ fusion protein was not degraded by lysosomes in rod photoreceptors in the absence of rhodopsin mislocalization (Ropelewski and Imanishi, 2019). Accelerated degradation of NKA $\alpha$ by lysosomes or proteasomes leads to its downregulation and exacerbates disease conditions such as damage of lung tissue or oxidative stress of kidney proximal tubule cells (Thévenod and Friedmann, 1999; Helenius et al., 2010). Likewise, the lysosomal pathway promotes NKA $\alpha$ degradation during rhodopsin mislocalization due to cotrafficking of IS PM-mislocalized rhodopsin with NKA $\alpha$ to lysosomes, serving as a common mechanism leading to neuronal dysfunction and degeneration (Ropelewski and Imanishi, 2019).

In summary, we demonstrated that RPE cells are capable of engulfing microvesicles released from rod photoreceptor cells. We believe that these vesicles are distinct from cilia-derived ectosomes or microvesicles described in peripherin/rds and PRCD (progressive rod-cone degeneration protein)-deficient mice (Salinas et al., 2017; Spencer et al., 2019). In these mouse models, there is no evidence of compromised rhodopsin entry into the cilia. Instead, continued entry of rhodopsin and other components is partly responsible for the bulging of the ciliary membrane and subsequent ectosome release. Moreover, RPE cells do not appear to be actively engulfing these ectosomes or microvesicles (Spencer et al., 2019). Class I mutant rhodopsin demonstrates compromised ciliary entry, similar to mouse models deficient in ciliary transport (Pazour et al., 2002). In these models, the IS PM, the site of rhodopsin accumulation, is the likely source of vesicles. This vesicle release is potentially a method to remove unwanted proteins during photoreceptor degeneration, when OS phagocytosis is compromised due to reduced OS transport of rhodopsin. Recent studies indicate that microvesicles are means of intercellular communication (Lee and Kim, 2017; Vidal-Gil et al., 2019). Contents of microvesicles, such as miRNA and signaling proteins, mediate phenotypic changes of the recipient cells (Schorey and Harding, 2016; Roballo et al., 2019). RPE cells undergo significant morphologic (Jones et al., 2016) and metabolic changes (Wang et al., 2016) in diseased retinas. In this study, we tracked microvesicles by fluorescently labeling them. This improved technique would be useful for identifying and purifying those photoreceptor-derived vesicles, which then would allow analysis of their components and further our understanding of intercellular communication in the pathologic retina.

\section{References}

Adams NA, Awadein A, Toma HS (2007) The retinal ciliopathies. Ophthalmic Genet 28:113-125.

Alfinito PD, Townes-Anderson E (2002) Activation of mislocalized opsin kills rod cells: a novel mechanism for rod cell death in retinal disease. Proc Natl Acad Sci U S A 99:5655-5660.

Blanks JC, Spee C (1992) Retinal degeneration in the pcd/pcd mutant mouse: accumulation of spherules in the interphotoreceptor space. Exp Eye Res 54:637-644.
Bonilha VL, Finnemann SC, Rodriguez-Boulan E (1999) Ezrin promotes morphogenesis of apical microvilli and basal infoldings in retinal pigment epithelium. J Cell Biol 147:1533-1548.

Concepcion F, Chen J (2010) Q344ter mutation causes mislocalization of rhodopsin molecules that are catalytically active: a mouse model of Q344ter-induced retinal degeneration. PLoS One 5: e10904.

Deretic D, Williams AH, Ransom N, Morel V, Hargrave PA, Arendt A (2005) Rhodopsin C terminus, the site of mutations causing retinal disease, regulates trafficking by binding to ADP-ribosylation factor 4 (ARF4). Proc Natl Acad Sci U S A 102:3301-3306.

Fowler CD (2019) NeuroEVs: characterizing extracellular vesicles generated in the neural domain. J Neurosci 39:9262-9268.

Friedrich $U$, Stöhr $H$, Hilfinger $D$, Loenhardt $T$, Schachner $M$, Langmann T, Weber BH (2011) The Na/K-ATPase is obligatory for membrane anchorage of retinoschisin, the protein involved in the pathogenesis of X-linked juvenile retinoschisis. Hum Mol Genet 20:1132-1142.

Hagstrom SA, Duyao M, North MA, Li T (1999) Retinal degeneration in tulp1-/- mice: vesicular accumulation in the interphotoreceptor matrix. Invest Ophthalmol Vis Sci 40:2795-2802.

Hagstrom SA, Adamian M, Scimeca M, Pawlyk BS, Yue G, Li T (2001) A role for the Tubby-like protein 1 in rhodopsin transport. Invest Ophthalmol Vis Sci 42:1955-1962.

Helenius IT, Dada LA, Sznajder JI (2010) Role of ubiquitination in Na, K-ATPase regulation during lung injury. Proc Am Thorac Soc 7:6570.

Hollingsworth TJ, Gross AK (2013) The severe autosomal dominant retinitis pigmentosa rhodopsin mutant Ter349Glu mislocalizes and induces rapid rod cell death. J Biol Chem 288:29047-29055.

Hu J, Bok D (2001) A cell culture medium that supports the differentiation of human retinal pigment epithelium into functionally polarized monolayers. Mol Vis 7:14-19.

Imanishi $Y$ (2019) Protein sorting in healthy and diseased photoreceptors. Annu Rev Vis Sci 5:73-98.

Jones BW, Pfeiffer RL, Ferrell WD, Watt CB, Marmor M, Marc RE (2016) Retinal remodeling in human retinitis pigmentosa. Exp Eye Res 150:149-165.

Kevany BM, Palczewski K (2010) Phagocytosis of retinal rod and cone photoreceptors. Physiology (Bethesda) 25:8-15.

Kokoska S, Zwillinger D (2000) CRC standard probability and statistics tables and formulae. Boca Raton, FL: Chapman \& Hall/CRC.

Kondo M, Sakai T, Komeima K, Kurimoto Y, Ueno S, Nishizawa Y, Usukura J, Fujikado T, Tano Y, Terasaki H (2009) Generation of a transgenic rabbit model of retinal degeneration. Invest Ophthalmol Vis Sci 50:1371-1377.

Kwok MC, Holopainen JM, Molday LL, Foster LJ, Molday RS (2008) Proteomics of photoreceptor outer segments identifies a subset of SNARE and Rab proteins implicated in membrane vesicle trafficking and fusion. Mol Cell Proteomics 7:1053-1066.

Laird JG, Pan Y, Modestou M, Yamaguchi DM, Song H, Sokolov M, Baker SA (2015) Identification of a VxP targeting signal in the flagellar $\mathrm{Na}+/ \mathrm{K}+$-ATPase. Traffic 16:1239-1253.

Lee JY, Kim HS (2017) Extracellular vesicles in neurodegenerative diseases: a double-edged sword. Tissue Eng Regen Med 14:667678.

Li T, Snyder WK, Olsson JE, Dryja TP (1996) Transgenic mice carrying the dominant rhodopsin mutation P347S: evidence for defective vectorial transport of rhodopsin to the outer segments. Proc Natl Acad Sci U S A 93:14176-14181.

Lodowski KH, Lee R, Ropelewski P, Nemet I, Tian G, Imanishi Y (2013) Signals governing the trafficking and mistrafficking of a ciliary GPCR, rhodopsin. J Neurosci 33:13621-13638.

Merin NM, Kelly KR (2014) Clinical use of proteasome inhibitors in the treatment of multiple myeloma. Pharmaceuticals (Basel) 8:120.

Molday LL, Wu WW, Molday RS (2007) Retinoschisin (RS1), the protein encoded by the X-linked retinoschisis gene, is anchored to the surface of retinal photoreceptor and bipolar cells through its 
interactions with a $\mathrm{Na} / \mathrm{K}$ ATPase-SARM1 complex. J Biol Chem 282:32792-32801.

Nagarajah S (2016) Exosome secretion-more than simple waste disposal? Implications for physiology, diagnostics and therapeutics. J Circ Biomark 5:7.

Nemet I, Tian G, Imanishi Y (2014) Submembrane assembly and renewal of rod photoreceptor cGMP-gated channel: insight into the actin-dependent process of outer segment morphogenesis. J Neurosci 34:8164-8174.

Nemet I, Ropelewski P, Imanishi Y (2015) Rhodopsin trafficking and mistrafficking: signals, molecular components, and mechanisms. Prog Mol Biol Transl Sci 132:39-71.

Nicholson C, Shah N, Ishii M, Annamalai B, Brandon C, Rodgers J, Nowling T, Rohrer B (2020) Mechanisms of extracellular vesicle uptake in stressed retinal pigment epithelial cell monolayers. Biochim Biophys Acta 1866:165608.

Nishimura DY, Fath M, Mullins RF, Searby C, Andrews M, Davis R, Andorf JL, Mykytyn K, Swiderski RE, Yang B, Carmi R, Stone EM, Sheffield VC (2004) Bbs2-null mice have neurosensory deficits, a defect in social dominance, and retinopathy associated with mislocalization of rhodopsin. Proc Natl Acad Sci U S A 101:1658816593.

Pan Y, Laird JG, Yamaguchi DM, Baker SA (2015a) A di-arginine ER retention signal regulates trafficking of $\mathrm{HCN} 1$ channels from the early secretory pathway to the plasma membrane. Cell Mol Life Sci 72:833-843.

Pan Y, Laird JG, Yamaguchi DM, Baker SA (2015b) An N-terminal ER export signal facilitates the plasma membrane targeting of HCN1 channels in photoreceptors. Invest Ophthalmol Vis Sci 56:35143521.

Pazour GJ, Baker SA, Deane JA, Cole DG, Dickert BL, Rosenbaum JL, Witman GB, Besharse JC (2002) The intraflagellar transport protein, IFT88, is essential for vertebrate photoreceptor assembly and maintenance. J Cell Biol 157:103-113.

Roballo KCS, da Silveira JC, Bressan FF, de Souza AF, Pereira VM, Porras JEP, Rós FA, Pulz LH, Strefezzi R. d F, Martins DDS, Meirelles FV, Ambrósio CE (2019) Neurons-derived extracellular vesicles promote neural differentiation of ADSCs: a model to prevent peripheral nerve degeneration. Sci Rep 9:11213.

Ropelewski P, Imanishi Y (2019) Disrupted plasma membrane protein homeostasis in a Xenopus laevis model of retinitis pigmentosa. J Neurosci 39:5581-5593.

Rückrich $T$, Kraus $M$, Gogel J, Beck A, Ovaa $H$, Verdoes $M$, Overkleeft HS, Kalbacher H, Driessen C (2009) Characterization of the ubiquitin-proteasome system in bortezomib-adapted cells. Leukemia 23:1098-1105.

Sakami S, Imanishi Y, Palczewski K (2019) Müller glia phagocytose dead photoreceptor cells in a mouse model of retinal degenerative disease. FASEB J 33:3680-3692.

Salinas RY, Pearring JN, Ding JD, Spencer WJ, Hao Y, Arshavsky VY (2017) Photoreceptor discs form through peripherin-dependent suppression of ciliary ectosome release. J Cell Biol 216:14891499.

Schneider BG, Kraig E (1990) Na+, K(+)-ATPase of the photoreceptor: selective expression of alpha 3 and beta 2 isoforms. Exp Eye Res 51:553-564.

Schneider CA, Rasband WS, Eliceiri KW (2012) NIH Image to ImageJ: 25 years of image analysis. Nat Methods 9:671-675.

Schön C, Asteriti S, Koch S, Sothilingam V, Garcia Garrido M, Tanimoto N, Herms J, Seeliger MW, Cangiano L, Biel M, Michalakis S (2016) Loss of HCN1 enhances disease progression in mouse models of CNG channel-linked retinitis pigmentosa and achromatopsia. Hum Mol Genet 25:1165-1175.

Schorey JS, Harding CV (2016) Extracellular vesicles and infectious diseases: new complexity to an old story. J Clin Invest 126:11811189.

Smith SJ, Fairclough L, Latinkic BV, Sparrow DB, Mohun TJ (2006) Xenopus laevis transgenesis by sperm nuclear injection. Nat Protoc 1:2195-2203.

Sparrow DB, Latinkic B, Mohun TJ (2000) A simplified method of generating transgenic Xenopus. Nucleic Acids Res 28:EXII.

Spencer WJ, Ding JD, Lewis TR, Yu C, Phan S, Pearring JN, Kim KY, Thor A, Mathew R, Kalnitsky J, Hao Y, Travis AM, Biswas SK, Lo WK, Besharse JC, Ellisman MH, Saban DR, Burns ME, Arshavsky VY (2019) PRCD is essential for high-fidelity photoreceptor disc formation. Proc Natl Acad Sci U S A 116:13087-13096.

Strauss $O$ (2005) The retinal pigment epithelium in visual function. Physiol Rev 85:845-881.

Sung CH, Makino C, Baylor D, Nathans J (1994) A rhodopsin gene mutation responsible for autosomal dominant retinitis pigmentosa results in a protein that is defective in localization to the photoreceptor outer segment. J Neurosci 14:5818-5833.

Thévenod F, Friedmann JM (1999) Cadmium-mediated oxidative stress in kidney proximal tubule cells induces degradation of $\mathrm{Na}+/$ $\mathrm{K}(+)$-ATPase through proteasomal and endo-/lysosomal proteolytic pathways. FASEB J 13:1751-1761.

Vidal-Gil L, Sancho-Pelluz J, Zrenner E, Oltra M, Sahaboglu A (2019) Poly ADP ribosylation and extracellular vesicle activity in rod photoreceptor degeneration. Sci Rep 9:3758.

Wang W, Lee SJ, Scott PA, Lu X, Emery D, Liu Y, Ezashi T, Roberts MR, Ross JW, Kaplan HJ, Dean DC (2016) Two-step reactivation of dormant cones in retinitis pigmentosa. Cell Rep 15:372-385.

Wetzel RK, Arystarkhova E, Sweadner KJ (1999) Cellular and subcellular specification of $\mathrm{Na}$,K-ATPase $\alpha$ and $\beta$ isoforms in the postnatal development of mouse retina. J Neurosci 19:9878-9889.

Wu YX, Yang JH, Saitsu H (2016) Bortezomib-resistance is associated with increased levels of proteasome subunits and apoptosisavoidance. Oncotarget 7:77622-77634.

Yau KW, Baylor DA (1989) Cyclic GMP-activated conductance of retinal photoreceptor cells. Annu Rev Neurosci 12:289-327.

Young RW (1967) The renewal of photoreceptor cell outer segments. J Cell Biol 33:61-72. 